\title{
A MAPS based pixel vertex detector for the STAR experiment
}

L. Greiner ${ }^{1}$, E. C. Anderssen, H.G. Ritter, J. Silber, T. Stezelberger, X. Sun, C. Q. Vu, H. H. Wieman

Lawrence Berkeley National Laboratory

1 Cyclotron Road, MS 70R0319, Berkeley, CA 94720, USA

E-mail: lcgreiner@lbl.gov

\section{J. Schambach}

University of Texas, Austin

1 University Station \#C1600, Austin, TX 78712, USA

E-mail: jschamba@physics.utexas.edu

\section{Szelezniak}

Institut Pluridisciplinaire Hubert Curien, 23 rue du Loess, 67037 Strasbourg, France

E-mail: michal.szelezniak@iphc.cnrs.fr

The STAR experiment at RHIC (Relativistic Heavy Ion Collider) is in the process of upgrading the inner detector region of the experiment to improve the vertex resolution. We describe the current design of a MAPS based vertex detector which is the innermost and highest resolution detector of the set of three planned upgrade detectors. This detector will enable the identification of decay vertices displaced from the interaction vertex by $100-150 \mu \mathrm{m}$ and extend the capabilities of the STAR detector in the heavy flavor domain. The detector is now a Department of Energy Nuclear Physics construction project and preliminary engineering production of limited set of detector ladders will begin in November of 2012. The production for the associated mechanical and electronics systems has already begun. We present selected detector design characteristics and prototyping results, which help to validate the design in preparation for the construction of the detector as well as first tests of the production prototype ladders, electronics and mechanical systems.

The 21st International Workshop on Vertex Detectors

16-21 September 2012

Jeju, Korea

Jeju, Korea

\footnotetext{
1 Speaker
} 


\section{Introduction}

We have completed the development phase and are entering the construction phase of a new vertex detector for the STAR experiment at the RHIC accelerator at BNL [1]. The primary physics requirement for this detector upgrade is to provide the vertex pointing capability to resolve vertices of $\mathrm{D}^{0}$ and other decays displaced from the primary interaction vertex by $100-150 \mu \mathrm{m}$ [2]. The PiXeL (PXL) detector is the innermost of 3 detector upgrades planned for the STAR experiment. The distance of closest approach (DCA) pointing resolution requirement of the system is approximately $30 \mu \mathrm{m}$ and is achieved by tracking inward from the Time Projection Chamber (TPC) through the new detector set with graded resolution. The stringent pointing resolution requirement leads to design goals for the PXL detector that include a radiation length of $<0.5 \%$ per layer and pixel positional stability to $20 \mu \mathrm{m}$. Sensors thinned to $50 \mu \mathrm{m}$, air cooling, very thin high modulus carbon fiber support structures and aluminum conductor readout cables are necessary constituents of the design. The final system design is an array of 40 ladders with ten $2 \mathrm{~cm} \times 2 \mathrm{~cm}$ sensors per ladder with parallel readout electronics chains for each ladder. We report on the characteristics of our design and the results of the development and prototype testing that we have accomplished in the critical areas of cooling, mechanical stability and readout cable development to validate this design. We also report on the characteristics of some of the production components.

\section{Detector requirements, characteristics, development and prototyping}

\subsection{PXL detector requirements and characteristics}

order to achieve the desired physics goals, the PXL detector has the following requirements:

- $-1 \leq \eta \leq 1$ and full azimuthal angle coverage (matching the TPC coverage).

- $\leq 30 \mu \mathrm{m}$ DCA pointing resolution required for $750 \mathrm{MeV} / \mathrm{c}$ kaons [2].

- Two or more layers with a separation of $>5 \mathrm{~cm}$.

- Pixel size of $\leq 30 \times 30 \mu \mathrm{m}^{2}$.

- Radiation length $\leq 0.5 \%$ / layer (including support structure). The goal is $0.37 \%$ / layer.

- $\quad$ 50-75 hits $/ \mathrm{cm}^{2}$ (inner layer) within the integration time of $200 \mu \mathrm{s}$ and at a Luminosity of $8 \times 10^{27} / \mathrm{cm}^{2} / \mathrm{s}$. (This requirement is based on Monte Carlo track reconstruction efficiency and is not a sensor or readout (RDO) limit)

- Survive a radiation environment at the (projected) level of 20 to $90 \mathrm{k}$ Rad /year and $2 \times 10^{11}-10^{12} / \mathrm{cm}^{2} 1 \mathrm{MeV} \mathrm{N}_{\mathrm{eq}} /$ year.

These requirements are based on extensive simulations and represent an optimization based on the physics goals and the STAR physical geometry. In order to meet these requirements, we have made choices that are reflected in our detector design. These design choices include:

- MAPS Pixel technology with a pixel size of $20.7 \mu \mathrm{m} \times 20.7 \mu \mathrm{m}$ and sensor power dissipation $\sim 170 \mathrm{~mW} / \mathrm{cm}^{2}$.

- Thinned silicon sensors (50 $\mu \mathrm{m}$ thickness).

- Air cooling.

- Quick extraction and detector replacement. 
The characteristics of this design include $356 \mathrm{M}$ pixels on $\sim 0.16 \mathrm{~m}^{2}$ of silicon, integration time of $185.6 \mu \mathrm{s}$ (to keep the pileup manageable), a DCA pointing resolution (separated into the geometrical and Multiple Coulomb Scattering components) of $12 \oplus 19 \mathrm{GeV} / \mathrm{p} \cdot \mathrm{c} \mu \mathrm{m}$, and a radiation length of $\mathrm{X} / \mathrm{X}_{0}=0.37 \%$ / layer. These characteristics along with the demonstrated radiation tolerance allow us meet the requiremetns needed to do the physics.

\subsection{PXL detector design}

The PXL detector design is as shown in Figure 1.

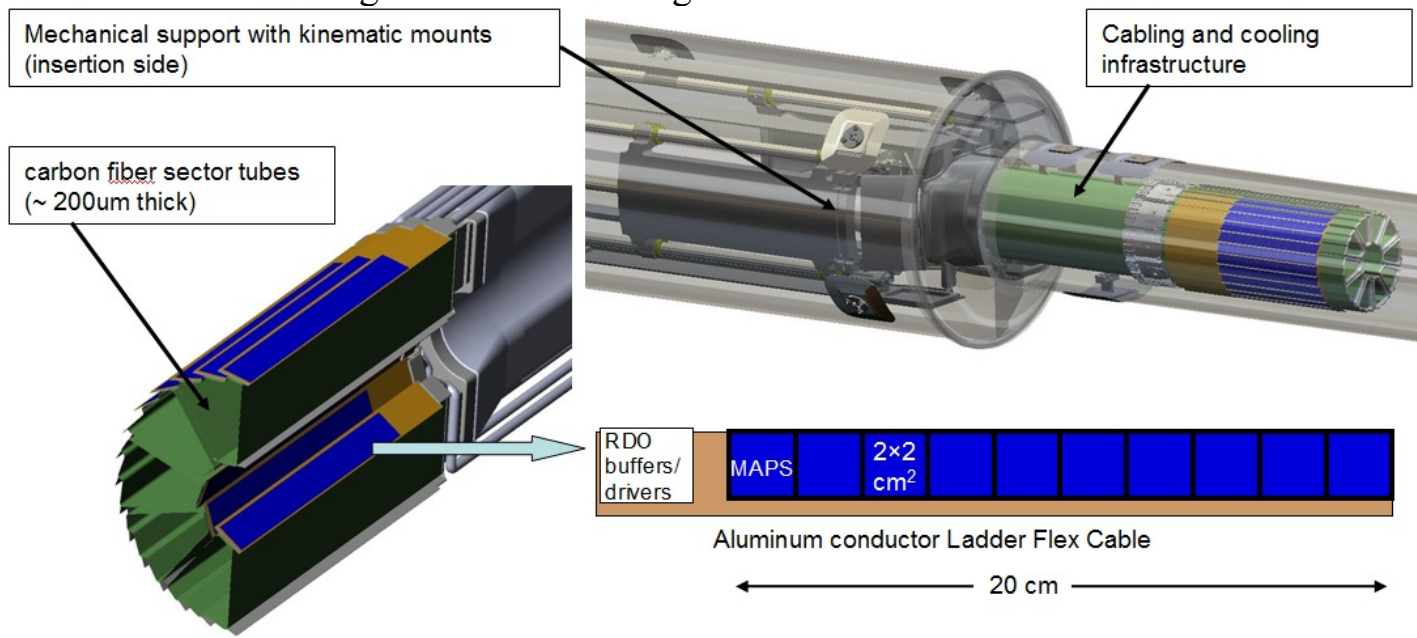

Figure 1: PXL detector design. The detector is composed of two half detectors, each with five sectors. Each sector is composed of three outer ladders and one inner ladder assembled onto a carbon fiber tube. Each ladder is composed of ten $\sim 2 \mathrm{~cm} \times \sim 2 \mathrm{~cm}$ MAPS sensors. The detector is supported and inserted from one side.

The PXL detector contains ten sectors arranged as a cylinder around the interaction point. Each sector is composed of a thin carbon fiber trapezoidal tube with three ladders of sensors attached to the outer surface and one ladder attached to the inner surface giving active tracking layers at $\mathrm{r}=2.5 \mathrm{~cm}$ and $\mathrm{r}=8 \mathrm{~cm}$. Each ladder contains ten $\sim 2 \mathrm{~cm}$ $x \sim 2 \mathrm{~cm}$ sensors. The detector is arranged into two detector halves with 5 sectors each that insert in parallel and close down around the beam pipe to provide hermetic coverage in phi. The detector is supported at one end which allows for a cart based insertion mechanism that guides the detector into location on low mass carbon fiber rails. The final position of the PXL detector is determined with high precision using kinematic mounts. This allows for quick detector insertion and replacement.

\subsection{PXL detector development and fabrication path}

The development and production path is geared toward two main deliverables. A prototype detector with at least 3 sectors will be fabricated with prototype sensors and copper conductor readout cables and is scheduled to be installed at RHIC for the 2013 run as an engineering prototype where the full detector function, mechanics, readout and interfaces to the existing STAR experiment will be tested. This limited production run is intended to develop the techniques and validate the tooling and assembly proceedures of ladder and sector production before the main production for the full detector taking 
place in the spring of 2013. The final production will deliver the full 10 sector system with production sensors and electronics to STAR for the 2014 run.

\subsection{Sensors}

The PXL detector can achieve several of its crucial characteristics, including high spatial resolution, substantial radiation hardness, and low material budget, by employing sensors developed in the Monolithic Active Pixel Sensors (MAPS) technology. We are using custom designed sensors developed by IPHC in Strasbourg, France [3]. The sensors that will be used in the PXL detector are designed as $928 \times 960$ pixel arrays of $20.7 \mu \mathrm{m}$ square pixels and have been fabricated in the $0.35 \mu \mathrm{m}$ Austria Micro Systems CMOS process. These sensors include on chip correlated double sampling, column level discriminators and a zero suppression system that provides hit pixel addresses. These addresses are read out by two Low-Voltage Differential Signaling (LVDS) outputs per sensor running at $160 \mathrm{MHz}$. The integration time of the sensor is $185.6 \mu \mathrm{s}$. The characteristics of this sensor architecture, including performance with a newly available high resistivity silicon process, are described in $[4,5,6]$. The first generation of these sensors has been fabricated and tested in high resistivity silicon and are performing as designed. The measured characteristics of the sensors produced include an efficiency of $>99 \%$ with an accidental noise hit rate of $<10^{-4}$ even after exposure to an ionizing radiation dose of $150 \mathrm{kRad}$ and non ionizing radiation dose of $3 \times 10^{12} 1 \mathrm{MeV} \mathrm{n}_{\mathrm{eq}} /$ $\mathrm{cm}^{2} \cdot[6]$

\subsection{Readout}

The thinned $50 \mu \mathrm{m}$ sensors will be mounted to two-layer $25 \mu \mathrm{m}$ Kapton flex cables with aluminum conductor to provide a readout path to the RDO system. The full data path is shown in Figure 2. 


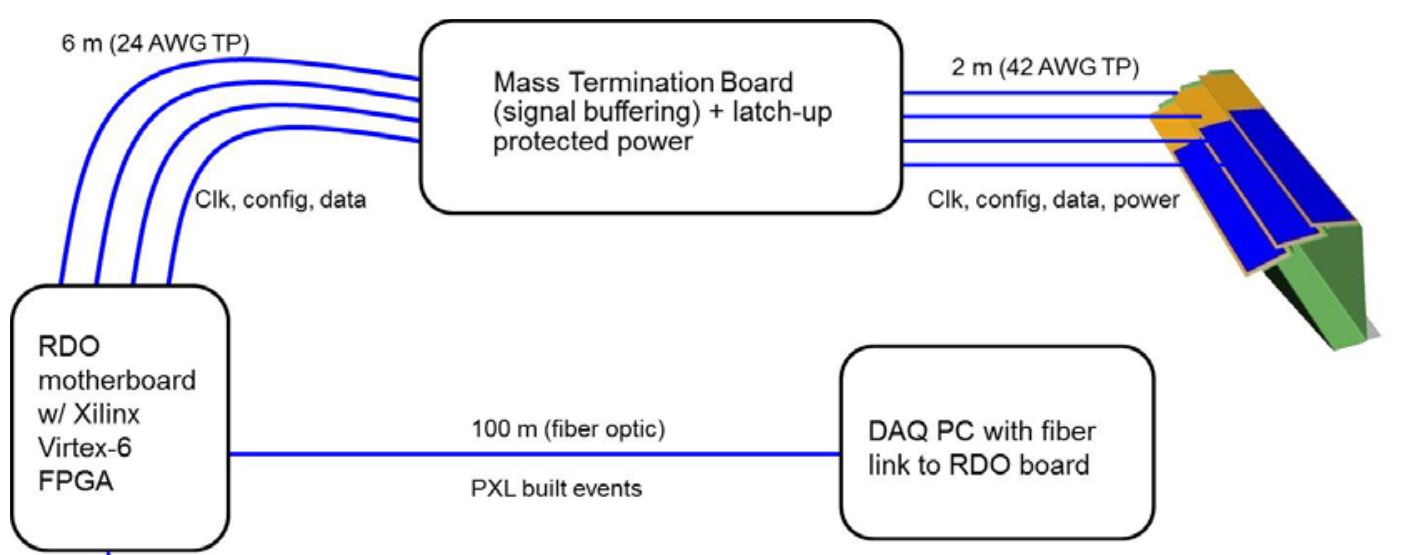

Highly parallel system

Trigger,

Slow control,

Configuration

etc.
- 4 ladders per sector

- 1 Mass Termination Board (MTB) per sector

- 1 RDO board per sector

- 10 RDO boards in the PXL system

Figure 2: PXL detector data path unit. Four ladders of sensors (one sector) are serviced by one MTB and one RDO motherboard. Latch-up protected power is regulated and supplied by daughter-cards located on the MTB. Data are formed into events in the RDO board and read out via fiber optic connections to data acquisition PCs. The PXL detector is composed of a parallel set of ten such units.

The PXL detector readout system is FPGA based and highly parallel, following the mechanical segmentation of the detector, with one sector (4 ladders) serviced by one Mass Termination Board (MTB) and RDO board. The complete detector is composed of a parallel set of ten such units. Signals to and from the sensors are buffered at the end of the ladder and out of the low mass region of the detector and carried over very fine twisted pair wires $(63 \mu \mathrm{m}$ diameter) to the MTB which provides additional buffering and drives the signals to the RDO board. The MTB also provides latch-up protected power to each ladder individually. Configuration and synchronization of the sensor ladders is handled by the RDO board which also receives triggers from the STAR trigger system and forms sensor data into an event structure. After initial configuration, the sensors are run continuously. Frame based data are received by the FPGA based RDO board, and on receipt of a level-0 trigger from the STAR trigger system, time selected hit data corresponding to a full frame are stored in memory. The event data are transferred to the STAR DAQ receiver PC over a high-speed bi-directional fiber link. We use the Source Interface Unit (SIU) and Readout Receiver Cards (RORC) [7] developed for the ALICE experiment at CERN as our optical link hardware to transfer data to and from the STAR data-acquisition system. Production prototypes of the MTB and RDO boards have been produced and are working as designed in our test system, which also includes a production prototype of a 10-sensor ladder. The pre-production RDO system has been used to read out prototype ladders at the full $160 \mathrm{MHz}$ readout speed and has been validated for all other functional readout requirements (configuration, slow control, trigger, etc.). 


\subsection{Probe testing}

We probe test thinned and diced sensors. This is necessary so that yield losses during the thinning and dicing process are not propagated into sensors installed on ladders where we require the full complement of 10 working sensors. Our system uses a 20 sensor vacuum chuck to hold the sensors in position on a probe testing machine where we do full functional testing including the JTAG configuration, determination of the threshold transfer functions and transition threshold points, the measurement and optimization of bias parameters, testing of the full readout path at $160 \mathrm{MHz}$, do an Light Emitting Diode (LED) flash to find dead and stuck pixels, test the zero suppression system with known patterns, measure the sensor noise, and measure the sensor response as a function of supply voltage. By performing this detailed testing regimen, our assembled ladders are formed with fully characterized and working sensors. The probe testing measurement of the sensor performance is consistent with the measurements obtained in the individual sensor testing done in reference [6]

\subsection{Cable and ladder}

The basic design for the readout cable is shown in the diagram below

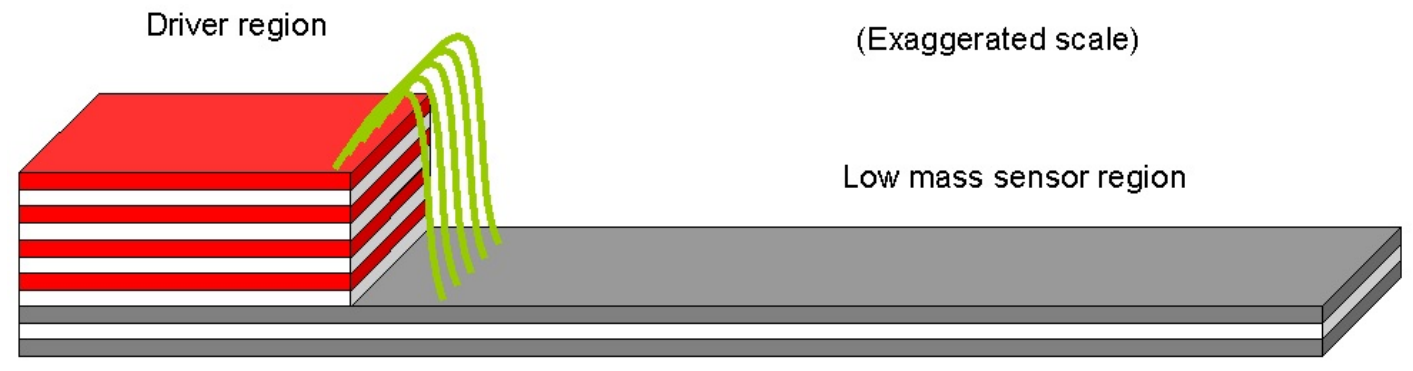

Figure 3: PXL ladder cable design. The cable is a hybrid design with a 2-layer aluminum conductor flex $P C B$ in the low mass sensor region mated to a copper conductor 4 layer flex PCB in the driver region. The connection between the cable types is made with bond wires.

This design has been extensively prototyped and tested and found to be fully functional. Based on this design, we have fabricated three pre-production prototype ladders to test our assembly procedures before entering production for the engineering run.

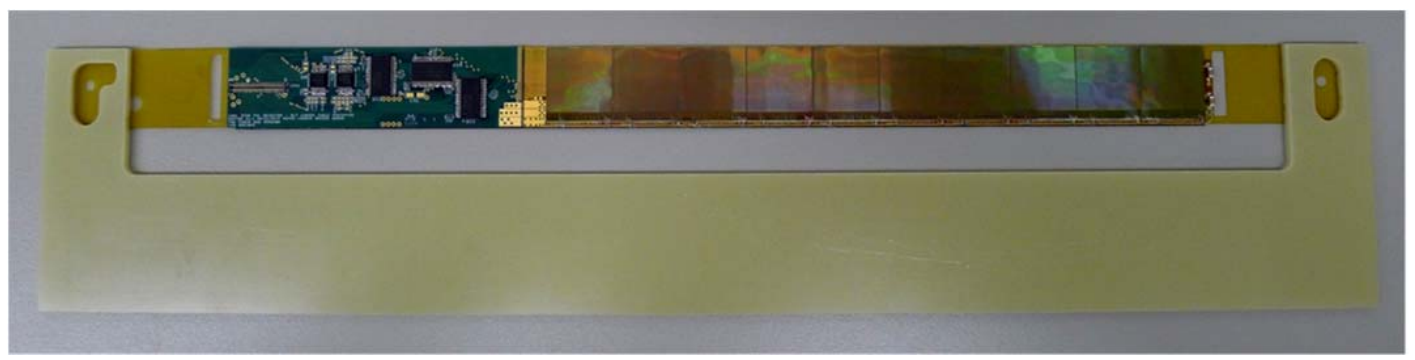

Figure 4: Production prototype ladder with 10 prototype sensors in the ladder configuration.

The results are very promising. The sensors mounted to ladders show the same amount of measured noise with all sensors running at the expected running occupancy, as the individual sensor measurements. With all sensors running into overflow on the zero 
suppression system (maximum data transfer and full digital activity in the sensors), the noise increases by only $\sim 25 \%$. This is the worst case scenario and not the normal running configuration. These results indicate that the ladder is working as designed and is acceptable for detector use.

\subsection{Mechanical systems}

The strict PXL radiation length requirement drives the mechanical design in several important ways. Air cooling and low mass support structures are required as is internal positional stability such that the intrinsic resolution of the detector is not compromised. We require a detector internal positional stability of $6 \mu \mathrm{m}$ RMS (20 $\mu \mathrm{m}$ envelope) while in operation and under cooling air flow. We have modeled the airflow and heat transfer capabilities of our prototype design as well as fabricated full scale detector mockups instrumented to allow for characterization of the sensor temperature and structure vibration as a function of cooling air velocity.

When the detector mockup is dissipating $340 \mathrm{~W}$ ( $270 \mathrm{~W}$ in the sensor region and $70 \mathrm{~W}$ in the driver region ) the test results show that with air cooling at $10 \mathrm{~m} / \mathrm{s}$ (mass flow of $\sim 0.42 \mathrm{~kg} / \mathrm{s}$ ) the temperature of the detector increases by approximately $12{ }^{\circ} \mathrm{C}$ above the ambient temperature. This is adequate to keep thermal distortions well below the required limit as well as keeping the sensors in a good operating temperature region. The measured vibration is within our required window of stability of a $6 \mu \mathrm{m}$ RMS for position. These measured results are consistent with the computational fluid dynamics simulations for the flow and thermal transfer characteristics.

Based on these successful tests we have completed the design for and constructed the full insertion mechanics for the PXL detector. The insertion mechanism was successfully tested both in the lab and at the STAR detector. A photograph of the insertion tests in the lab is shown as Figure 5.

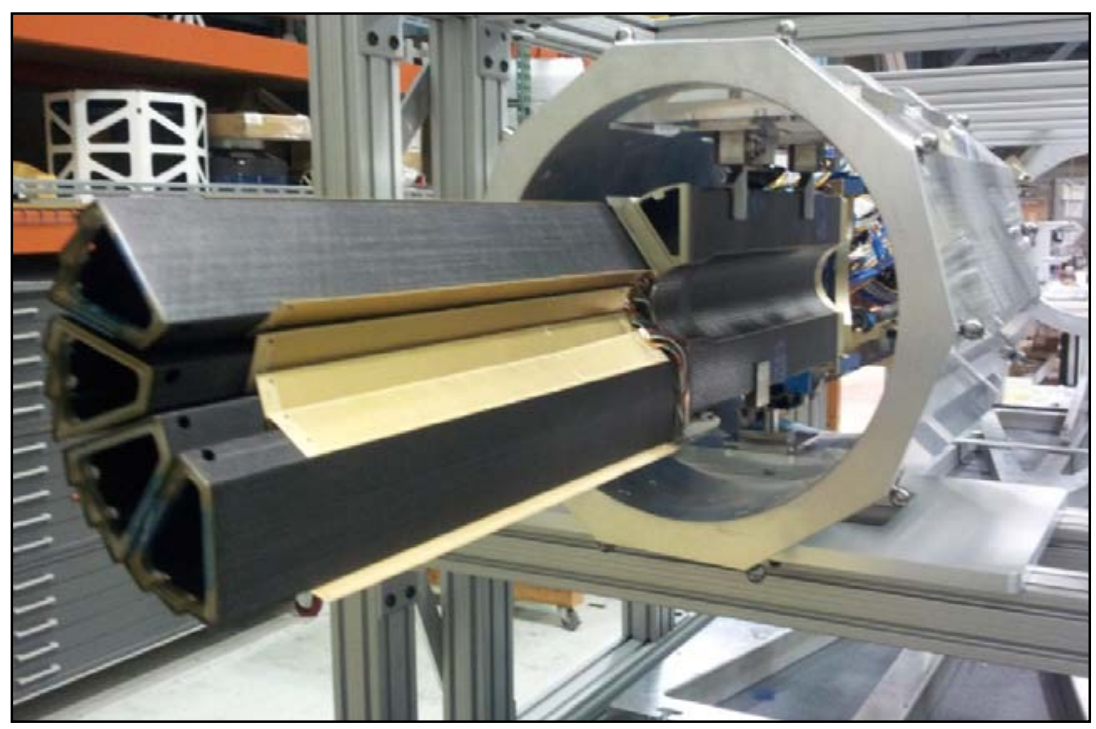

Figure 5 Insertion test of the STAR PXL detector at the lab at LBNL. Note the kinematic mounts used for detector positioning, two at the top and one on the bottom. 


\section{Summary and Plans}

The STAR PXL detector is about to enter production and is scheduled to be used in the 2014 STAR heavy ion run at RHIC. This detector is the culmination of a lengthy development and testing process and represents the first large scale use of MAPS sensors in a tracking detector at a collider experiment. The results from an extensive testing program indicate that we should be able to build a detector with very low radiation length and high spatial vertex resolution (DCA) for use in the heavy quark physics program at RHIC.

\section{Acknowledgements}

We gratefully acknowledge Marc Winter and the IPHC group for the development of the PXL detector sensors. This work received support in part from the US Department of Energy Contract no. DE-AC02-05CH11231, Office of Nuclear Physics.

\section{References}

[1] J. Adams et al., "Experimental and theoretical challenges in the search for the quark-gluon plasma: The STAR Collaboration's critical assessment of the evidence from RHIC collisions", Nucl. Phys. A 757 (2005), 102-183.

[2] C. Chasman et al., "A Heavy Flavor Tracker for STAR”, (2008), LBNL/PUB-5509-2008.

[3] M.Winter, "Achievements and perspectives of CMOS pixel sensors for charged particle tracking”, Nucl. Instrum. Meth. A 623 (2010), 192-194, doi:10.1016/j.nima.2010.02.192

[4] C. Hu-Guo et al., "First reticule size MAPS with digital output and integrated zero suppression for the EUDET-JRA1 beam telescope", Nucl. Instrum. Meth. A 623 (2010), 480-482, doi:10.1016/j.nima.2010.03.043

[5] A. Dorokhov et al., "High-resistivity CMOS pixel sensors and their application to the STAR PIXEL detector”, Nucl. Instrum. Meth. A 650 (2011), 174-177, doi: 10.1016/j.nima.2010.12.112

[6] Valin et al., A reticle size CMOS pixel sensor dedicated to the STAR HFT, JINST 7 (2012) C01102

[7] http://alice-proj-ddl.web.cern.ch/alice-proj-ddl/ 